\title{
COVERING OF A HOLOMORPHICALLY CONVEX MANIFOLD CARRYING A POSITIVE LINE BUNDLE
}

\author{
SAÏD ASSERDA
}

Let $M$ be a holomorphically convex manifold and $\pi: \tilde{M} \rightarrow$ $M$ is a holomorphic connected covering. If $M$ carries a positive holomorphic line bundle $L$ such that the cohomology class of $\pi^{*} L$ in $H^{1}\left(\tilde{M}, \mathcal{O}^{*}\right)$ vanishes, then $\tilde{M}$ is a Stein manifold.

A classical theorem of Siegel $[\mathbf{S}]$ asserts that a bounded domain in $\mathbf{C}^{n}$ covering a compact complex manifold is a domain of holomorphy. In [Wa] Watanabe showed that if a complex manifold $D$ covering a projective manifold $M$ and satisfies $H^{1}\left(D, \mathcal{O}^{*}\right)=0$, then $D$ is a Stein manifold with $H^{2}(D, \mathbf{Z})=0$, where $\mathcal{O}^{*}$ is the sheaf of germs of nowhere-vanishing holomorphic functions and $\mathbf{Z}$ is the additive group of integers. The purpose of this paper is to study the case where the base of covering is a holomorphically convex complex manifold carrying a positive holomorphic line bundle. Recall that a complex manifold $M$ is holomorphically convex if, for every infinite subset $S$ of $M$ without limit points, there is a holomorphic function $f$ on $M$ which is unbounded on $S$. Our main result is the following:

Theorem. Let $M$ be a holomorphically convex complex manifold and $\pi: \tilde{M} \rightarrow M$ is a holomorphic connected covering. If $M$ carries a positive holomorphic line bundle $L$ such that the cohomology class of $\pi^{*} L$ in $H^{1}\left(\tilde{M}, \mathcal{O}^{*}\right)$ vanishes, then $\tilde{M}$ is a Stein manifold.

It is well known that there is an isomorphism between the class of holomorphic line bundles over a complex manifold $X$ and the cohomology group $H^{1}\left(X, \mathcal{O}^{*}\right)$ (see [We], Lemma 4.4, p. 101). The cohomology class of a line bundle $F$ is defined as the class in $H^{1}\left(X, \mathcal{O}^{*}\right)$ of a holomorphic cocycle $\left\{f_{i j}\right\}$ representing $F$. The vanishing of the cohomology class of $\pi^{*} L$ in $H^{1}\left(\tilde{M} \mathcal{O}^{*}\right)$ is necessary as shown later. Since a compact complex manifold is vacuously holomorphically convex, Watanabe's theorem is a consequence of our theorem. But in his proof, it is not clear that the strongly plurisubharmonic function $\phi$ ([Wa], p. 244) does not grow to $-\infty$ near the topological boundary of $\tilde{M}$. Also as remarked by him, the condition $H^{1}\left(\tilde{M} \mathcal{O}^{*}\right)=0$ cannot 
be replaced by $H^{1}(\tilde{M}, \mathcal{O})=0$, where $\mathcal{O}$ is the sheaf of germs of holomorphic functions: Consider the case $M=\tilde{M}=\mathbf{P}_{1}(C)$ and $\pi$ is the identity mapping.

Proof of theorem. As in [Wa] let $\left\{V_{i}\right\}$ be an open covering of $M$ such that each $V_{i}$ is a local coordinate neighborhood and is biholomorphic to a connected component $\pi^{-1}\left(V_{i}\right)$. Choosing a suitable refinement $\left\{U_{i}\right\}$ of $\left\{V_{i}\right\}$, we can represent the line bundle $L$ by a system of transition functions $\left\{f_{i j}\right\}$ and find a Hermitian metric $\left\{a_{j}\right\}$ along the fibers of $L$ which satisfies the following conditions:

(i) Each $a_{j}$ is a $C^{\infty}$, real valued and positive function on $U_{j}$.

(ii) If $U_{j} \cap U_{k}=\emptyset$, then we have $a_{k}=\left|f_{j k}\right|^{2} a_{j}$.

(iii) The function $-\log a_{j}$ is strongly plurisubharmonic on $U_{j}$ i.e. the

$$
(1,1) \text {-form } i \partial \bar{\partial} \log \frac{1}{a_{j}} \text { is positive definite. }
$$

The curvature of $L$ is defined as $c(L):=i \partial \bar{\partial}\left(-\log a_{j}\right)$ on $U_{j} . L$ is said to be positive if $c(L)$ is a positive definite $(1,1)$-form on the convex tangent bundle $T M$.

The line bundle $\pi^{*} L$ defined on $\tilde{M}$ has $\left\{f_{i j} o \pi\right\}$ as transition functions and $\left\{a_{j} o \pi\right\}$ for Hermitian metric. Since $\left\{\pi^{-1}\left(U_{j}\right)\right\}$ is an open covering of $\tilde{M}$, $\left\{f_{i j} o \pi\right\}$ defines an element of the cohomology class of $\pi^{*} L$. By hypothesis $\left[\pi^{*} L\right]=0$ in $H^{1}\left(\tilde{M}, \mathcal{O}^{*}\right)$, then there an open covering $Y_{j}$ of $\tilde{M}$ such that the cohomology class of $\left[f_{i j} o \pi\right]=0$ in $H^{1}\left(\left\{Y_{j}\right\}, \mathcal{O}^{*}\right)$. Taking a refinment of $\left\{U_{j}\right\}$ and $\left\{Y_{j}\right\}$, we may suppose that $\left\{U_{j}\right\}=\left\{Y_{j}\right\}$. Then there is a cochain $\left\{f_{j}\right\}$ of $C^{0}\left(\left\{\pi^{-1}\left(U_{j}\right)\right\}, \mathcal{O}^{*}\right)$ such that $f_{j k} O \pi=\frac{f_{k}}{f_{j}}$ on $\pi^{-1}\left(U_{j}\right) \cap \pi^{-1}\left(U_{k}\right)$. The holomorphic section of $\pi^{*} L$ over $\tilde{M}$ defined by $s=f_{j}$ on $\pi^{*}\left(U_{j}\right)$ is nowhere zero. We can define a $C^{\infty}$ strongly Psh function on $\tilde{M}$ in the following way:

$$
\phi(x):=-\log \left(a_{j} o \pi(x)\left|f_{j}(x)\right|^{2}\right)=-\log \|s(x)\|_{\pi^{*} L}^{2} \quad \text { for } \quad x \in \pi^{-1}\left(U_{j}\right)
$$

where $\|s\|_{\pi^{*} L}$ is the norm of $s$ with respect to the Hermitian metric of $\pi^{*} L$ induced by $L$.

Since $M$ is holomorphically convex and $L$ is positive, it is easy to see that $M$ admits a complete Kähler metric $g$. Simply take

$$
g:=i \partial \bar{\partial}(\chi o \Psi)+c(L)
$$

where $\Psi \in C^{\infty}(M)$ is an exhaustive Psh function (see $[\mathbf{H}]$, p. 117, Theorem 5.1.6) and $\chi: \mathbf{R} \rightarrow \mathbf{R}$ is a smooth function such that $\chi^{\prime}>0, \chi^{\prime \prime} \geq 0$, and $\chi^{\prime}(t) \rightarrow+\infty$ fast as $t \rightarrow+\infty$. Since $\pi$ is a connected covering, the pull back $\pi^{*} g$ of $g$ by $\pi$, define a complete Kähler metric $\tilde{g}$ on $\tilde{M}$. 
Now let $S$ be an infinite subset without linit point in $\tilde{M}$. We must produce a holomorphic function $f$ on $\tilde{M}$ such that $|f|$ is unbounded on $S$. Since it suffices to consider any infinite subset of $S$, we may assume that $S$ is actually equal to a sequence of points $\left\{x_{\nu}\right\}$.

Case 1. If $\left\{y_{\nu}\right\}$ has a limit point $y$ in $M$, then we may assume $y_{\nu} \rightarrow y$. Let $(U \phi)$ be a local coordinate neighborhood such that $\phi(y)=0, \phi(U)=$ $B(0, R) \subset \mathbf{C}^{n}$ and $\pi^{-1}(U)=\cup_{j} V_{j}$ with $V_{j} \cap V_{k}=\emptyset$ for every $j \neq k$ and $\pi_{j}:=\left.\pi\right|_{V_{j}}: V_{j} \rightarrow U$ is a biholomorphic map. Taking $\nu$ large enough, we may assume that $y_{\nu} \in U$ for $\nu=1,2, \ldots$. Since $x_{\nu} \in \pi^{-1}(U)$ there is a unique $j(\nu) \in \mathbf{N}$ such that $x_{\nu} \in V_{j(\nu)}$. If $I_{\nu}:=\{\nu \in \mathbf{N}, j(\nu)=j(\mu)\}$ and $\nu_{k}=\sup \left\{\nu \in I_{k}\right\}$, then $x_{\nu_{k}} \in V_{\nu_{k}}$ and $V_{j(k)} \cap V_{j\left(k^{\prime}\right)}=\emptyset$ if $k \neq k^{\prime}$. Since the set $I_{k}$ is finite, the subsequence $\left\{z_{k}:=x_{\nu_{k}}\right\}$ has not limit point in $\tilde{M}$. Set $W_{k}:=V_{j\left(\nu_{k}\right)}$ and $\pi_{k}:=\pi_{j\left(\nu_{k}\right)}$ and consider the biholomorphic map $\phi_{k}=\phi o \pi_{k}: W \rightarrow B(0, R)$. The map $\phi_{k}$ satisfies the following properties:

(1) $\phi_{k}\left(z_{k}\right)=\phi\left(y_{\nu_{k}}\right) \forall k \in \mathbf{N}$; and

(2) $\alpha \phi_{k}^{*} g_{e} \leq \tilde{g} \leq \beta \phi_{k}^{*} g_{e}$ on $W_{k}$ where the constants $\alpha, \beta$ are independent of $k$ and $g_{e}$ is the Euclidean metric of $\mathbf{C}^{n}$.

Without loss of generality, we may assume that $\left\|\phi_{k}\left(z_{k}\right)\right\| \leq \frac{R}{9}$. Hence $B\left(0, \frac{R}{9}\right) \subset \subset B\left(\phi_{k}\left(z_{k}\right), \frac{R}{9}\right) \subset \subset B(0, R)$ and by (2) we have

$$
\alpha\left\|\phi_{k}(x)-\phi_{k}\left(z_{k}\right)\right\| \leq d_{\tilde{g}}\left(x, z_{k}\right) \leq \beta\left\|\phi_{k}(x)-\phi_{k}\left(z_{k}\right)\right\| \quad \forall_{x} \in W_{k} .
$$

Thus $X_{k}:=\phi^{-1}\left(B\left(\phi_{k}\left(z_{k}\right), \frac{R}{4}\right)\right) \subset B_{\tilde{g}}\left(z_{k}, \frac{\beta B}{4}\right)$. Let $\lambda$ be a test function in $B\left(0, \frac{B}{8}\right)$ such that $\lambda=1$ in $B\left(0, \frac{R}{9}\right)$. Let $\Psi: \tilde{M} \rightarrow \mathbf{R} \cup\{-\infty\}$ be the function $\Psi$ defined by

$$
\Psi(x)= \begin{cases}\lambda\left(\phi_{k}(x)\right) \log \left\|\phi_{k}(x)-\phi_{k}\left(z_{k}\right)\right\|^{2 n} & \text { if } x \in X_{k} \\ 0 & \text { if } x \in \tilde{M} \backslash \cup_{k} X_{k} .\end{cases}
$$

Then $\Psi$ is a smooth function on $\tilde{M} \backslash\left\{z_{k}\right\}_{k=1}^{\infty}$. Thanks to (2) there is a constant $K>0$ such that

$$
i \partial \bar{\partial} \Psi \geq-K \tilde{g} \text { in a distributional sense on all of } \tilde{M} \text {. }
$$

Put $\gamma_{k}=\sup _{X_{k}}\|s\|_{\pi^{*} L}^{2}$ and $\rho_{k}=\sup _{1<j<k} \gamma_{j}$. By passing to a subsequence, we may assume that $r\left(z_{k}\right):=d_{\tilde{g}}\left(z_{k}, x_{o}\right) \geq k+\rho_{k}$ for all $k \in \mathbf{N}\left(x_{o} \in \tilde{M}\right.$ is a fixed point).

Lemma ([N1, Lemma 1.1]). There is a smooth and exhaustive function $\tau: \tilde{M} \rightarrow \mathbf{R}$ such that

(i) $r \leq C_{1} \tau \leq C_{2} r$; and

(ii) $i \partial \bar{\partial} \tau \geq-C_{3} \tilde{g}$, on $\pi^{-1}(U)$ 
where the constants may depend on $U$.

Define a smooth section $t$ of $\pi^{*} L^{m}$ on $\tilde{M}$ by

$$
t(x)= \begin{cases}\lambda\left(\phi_{k}(x)\right) e^{r\left(z_{k}\right)} \bigotimes_{1}^{m} s(x) & \text { if } x \in X_{k} \\ 0 & \text { elsewhere }\end{cases}
$$

We obtain a smooth $\bar{\partial}$-form of type $(0,1)$ with values in $\pi^{*} L^{m}$ and support contained in $\cup_{k=1}^{\infty} X_{k}$, by defining

$$
\omega=\bar{\partial} t
$$

Since $\lambda=1$ on $B\left(0, \frac{R}{8}\right)$ and $s$ is holomorphic, we conclude that $\omega$ vanishes on $\phi_{k}^{-1}\left(B\left(0, \frac{R}{8}\right)\right) \subset X_{k}$ for $k=1,2,3, \ldots$. Moreover, we have $\left|\lambda o \phi_{k}\right| \leq$ Const on $X_{k} \backslash \phi_{k}^{-1}\left(B\left(0, \frac{R}{8}\right)\right)$, and, because the map $\phi_{k}$ satisfies the property (2), we have $\left|\bar{\partial}\left(\lambda o \phi_{k}\right)\right| \leq$ Const on $X_{k}$, where the constants are independent of $k$. Therefore on the set $X_{k} \backslash \phi_{k}^{-1}\left(B\left(0, \frac{R}{8}\right)\right)$, hence on all $X_{k}$, we have

$$
\|\omega\|_{e^{-\Psi} \pi^{*} L^{m}} \leq \text { Const } e^{2 r\left(z_{k}\right)} \rho_{k}^{2 m}
$$

where the constant is independent of $k$. Also as mentioned above, $\omega$ vanishes on the complement $\tilde{M} \backslash \cup_{k} X_{k}$. Therefore, if we define a singular Hermitian metric in $\pi^{*} L^{m}$ on $\tilde{M}$ by

$$
\|?\|_{m}^{2}:=e^{-\left(3 C_{1} r+\Psi\right)}\|?\|_{\pi^{*} L^{m}}^{2}
$$

then $\|?\|_{m}$ is smooth on $\tilde{M} \backslash\left\{z_{k}\right\}_{k=1}^{\infty}$, and

$$
\begin{aligned}
\int_{\tilde{M}}\|\omega\|_{m}^{2} d V_{\tilde{g}} & =\int_{\tilde{M}}\|\omega\|_{e^{\Psi} \pi^{*} L^{m}}^{2} e^{-3 C_{1} r} d V_{\tilde{g}} \\
& =\sum_{k=1}^{\infty} \int_{X_{k}}\|\omega\|_{e^{\Psi} \pi^{*} L^{m}}^{2} e^{-3 C_{1} r} d V_{\tilde{g}}
\end{aligned}
$$

Hence

$$
\int_{\tilde{M}}\|\omega\|_{m}^{2} d V_{\tilde{g}} \leq \text { Const } \sum_{k=1}^{\infty} \rho_{k}^{2 m} e^{2 r\left(z_{k}\right)} e^{-3 C_{1} r} d V_{\tilde{g}} .
$$

By the previous Lemma, we have $r \leq C_{1} r$ om $X_{k}$. Therefore, since $X_{k} \subset$ $B_{\tilde{g}}\left(z_{k}, \frac{\beta R}{4}\right)$,

$$
r\left(z_{k}\right)-\frac{\beta R}{4} \leq r(x) \leq C_{1} r(x)
$$


for every $x \in X_{k}$ and $k=1,2, \ldots$. Thus

$$
\begin{aligned}
\int_{\tilde{M}}\|\omega\|_{m}^{2} d V_{\tilde{g}} & \leq \text { Const } \sum_{k=1}^{\infty} \rho_{k}^{2 m} \int_{X_{k}} e^{2 r\left(z_{k}\right)-3 r\left(z_{k}\right)} d V_{\tilde{g}} \\
& \leq \sum_{k=1}^{\infty} \rho_{k}^{2 m} e^{-r\left(z_{k}\right)} \operatorname{vol}\left(B_{g_{e}}\left(0, \frac{R}{4}\right)\right) \\
& \leq \text { Const } \sum_{k=1}^{\infty} \rho_{k}^{2 m} e^{-\rho_{k}} e^{-k}
\end{aligned}
$$

Let $a:=\lim _{k \rightarrow \infty} \rho_{k}$. If $a<+\infty$ then

$$
\int_{\tilde{M}}\|\omega\|_{m}^{2} d V_{\tilde{g}} \leq \text { Const } \sum_{k=1}^{\infty} e^{-k}<+\infty
$$

Now if $a=+\infty$, then

$$
\int_{\tilde{M}}\|\omega\|_{m}^{2} d V_{\tilde{g}} \leq \text { Const } \sum_{k=1}^{\infty} \rho_{k}^{2 m} e^{-\rho_{k}}<+\infty .
$$

Let $f$ be a smooth $(0,1)$-form in $\tilde{M}$ with values in $\pi^{*} L$. By Cauchy-Schwarz inequality

$$
\left(\int_{\tilde{M}}\left|\langle f, \omega\rangle_{m}\right| d V_{\tilde{g}}\right)^{2} \leq \int_{\tilde{M}}\|\omega\|_{m}^{2} d V_{\tilde{g}} \int_{\operatorname{supp} t(\omega)}\|f\|_{m}^{2} d V_{\tilde{g}}
$$

There exist a constants $c>0$ and $d$ such that $c(L) \geq c . g$ and $\operatorname{Ricci}(g) \geq d . g$ on $U$. Since $c\left(\pi^{*}\left(L^{m}\right)\right)=m \pi^{*}(c(L))$, for $m$ sufficiently large we have

$$
\operatorname{Ricci}(\tilde{g})+i \partial \bar{\partial} \Psi+i 3 C_{1} \partial \bar{\partial}+m \pi^{*} c(L) \geq \tilde{g} \quad \text { on } \pi^{-1}(U) .
$$

Using the Bochner-Kodaira-Nakano equality in Kählerian geometry [D], we have

$$
\begin{aligned}
& \left(\int_{\tilde{M}}\left|\langle f, \omega\rangle_{m}\right| d V_{\tilde{g}}\right)^{2} \\
& \leq \int_{\tilde{M}}\|\omega\|_{m}^{2} d V_{\tilde{g}}\left(\int_{\operatorname{supp} t(\omega)}\left\|\overline{\partial^{*}} f\right\|_{m}^{2} d V_{\tilde{g}}+\int_{\operatorname{supp} t(\omega)}\|\bar{\partial} f\|_{m}^{2} d V_{\tilde{g}}\right),
\end{aligned}
$$

where $\overline{\partial^{*}}$ is the formal adjoint of $\bar{\partial}$ acting on $(0,1)$-forms in $L^{2}\left(\tilde{M} ;\left(\pi^{*} L^{m}\right.\right.$, $\left.\left.\|?\|_{m}\right)\right)$ as an unbounded operator. Since the metric $\tilde{g}$ is complete, the space of smooth $(0,1)$-forms with compact support on $\tilde{M}$ and with values in $\pi^{*} L^{m}$ is dense in $\operatorname{Dom}\left(\overline{\partial^{*}}\right) \cap \operatorname{Ker} \bar{\partial}$ with respect to the (singular) graph norm $[\mathbf{D}]$ :

$$
\|f\|:=\int_{\tilde{M}}\|f\|_{m}^{2} d V_{\tilde{g}}+\int_{\tilde{M}}\left\|\overline{\partial^{*}} f\right\|_{m}^{2} d V_{\tilde{g}}+\int_{\tilde{M}}\|\bar{\partial} f\|_{m}^{2} d V_{\tilde{g}}
$$


By density, $\forall f \in \operatorname{Dom}\left(\overline{\partial^{*}}\right) \cap \operatorname{Ker} \bar{\partial}$ we have

$$
\begin{aligned}
& \int_{\tilde{M}}\left|\langle f, \omega\rangle_{m}\right| d V_{\tilde{g}} \\
& \leq\left(\int_{\tilde{M}}\|\omega\|_{m}^{2} d V_{\tilde{g}}\right)^{\frac{1}{2}}\left(\int_{\tilde{M}}\left\|\overline{\partial^{*}} f\right\|_{m}^{2} d V_{\tilde{g}}+\int_{\tilde{M}}\|\bar{\partial} f\|_{m}^{2} d V_{\tilde{g}}\right)^{\frac{1}{2}} .
\end{aligned}
$$

Since $\bar{\partial} \omega=0$, it suffices to consider the $\bar{\partial}$-closed forms $f$. The above inequality become

$$
\begin{aligned}
& \int_{\tilde{M}}\left|\langle f, \omega\rangle_{m}\right| d V_{\tilde{g}} \\
& \leq\left(\int_{\tilde{M}}\|\omega\|_{m}^{2} d V_{\tilde{g}}\right)^{\frac{1}{2}}\left(\int_{\tilde{M}}\left\|\overline{\partial^{*}} f\right\|_{m}^{2} d V_{\tilde{g}}\right)^{\frac{1}{2}} \quad \forall f \in \operatorname{Dom} \overline{\partial^{*}}
\end{aligned}
$$

By Lemma 4.1.1 in $[\mathbf{H}]$, there exists a smooth section $\sigma$ of $\pi^{*} L^{m}$ on $\tilde{M}$ such that

$$
\bar{\partial} \sigma=\omega \quad \text { and } \quad \int_{\tilde{M}}\|\sigma\|_{m}^{2} d V_{\tilde{g}} \leq \int_{\tilde{M}}\|\omega\|_{m}^{2} d V_{\tilde{g}} .
$$

Therefore $\|\sigma\|_{m}^{2}$ is integrable on the neighborhood $\phi_{k}^{-1}\left(B\left(0, \frac{R}{8}\right)\right)$ of $z_{k}$, on which

$$
\Psi(x)=n \log \left\|\phi_{k}(x)-\phi_{k}\left(z_{k}\right)\right\|^{2} .
$$

Hence

$$
\begin{aligned}
\infty & >\int_{\phi_{k}^{-1}\left(B\left(0, \frac{R}{8}\right)\right)}\|\sigma\|_{m}^{2} d V_{\tilde{g}} \\
& =\int_{\phi_{k}^{-1}\left(B\left(0, \frac{R}{8}\right)\right)}\|\sigma\|_{\pi^{*} L^{m}} e^{-3 C_{1} r} e^{-\Psi} d V_{\tilde{g}} \\
& =\int_{\phi_{k}^{-1}\left(B\left(0, \frac{R}{8}\right)\right)} \frac{\|\sigma\|_{\pi^{*} L^{m}}^{2} e^{-3 C_{1} r}}{\left\|\phi_{k}-\phi_{k}\left(z_{k}\right)\right\|^{2 n}} d V_{\tilde{g}} .
\end{aligned}
$$

However $\|\sigma(x)\|_{\pi^{*} L^{m}}^{2} e^{-3 C_{1} r(x)}$ is smooth while $\left\|\phi_{k}-\phi_{k}\left(z_{k}\right)\right\|^{-2 n}$ is not locally integrable at $z_{k}$, since $\tilde{M}$ has real dimension $2 n$ and $\phi_{k}\left(z_{k}\right)=0$. It follows that

$$
\sigma\left(z_{k}\right)=0
$$

for $k=1,2,3, \ldots$. If $f$ is the holomorphic function defined on $\tilde{M}$ in the following way:

$$
f:=\frac{\sigma}{\otimes_{1}^{m} s},
$$

then $f\left(z_{k}\right)=e^{r\left(z_{k}\right)} \rightarrow \infty$ as $k \rightarrow \infty$. It follows that $f$ is unbounded on $S$. 
Case 2. If $\left\{y_{\nu}\right\}$ has no limit point in $M$, then there exists a holomorphic function $f$ on $M$ such that $\left|f\left(y_{\nu}\right)\right|$ is unbounded. Hence we may take $\pi^{*} f$ as our desired holomorphic function on $\tilde{M}$.

Since $\tilde{M}$ supports a smooth strongly plurisubharmonic function, then $\tilde{M}$ is a Stein manifold.

As mentioned in the introduction, the condition $\left[\pi^{*} L\right]=0$ in $H^{1}\left(\tilde{M}, \mathcal{O}^{*}\right)$ is necessary. We give here an example $\tilde{M}$ of a covering of a smooth projective manifold $M$ which is not holomorphically convex and for every positive line bundle $L$ over $M$, the cohomology class of $\pi^{*} L$ is not zero in $H^{1}\left(\tilde{M}, \mathcal{O}^{*}\right)$. An example of $M$ such that $\tilde{M}$ is not holomorphically convex is given in [N2], Ex. 4, 5, p. 451. We will use it to verify the nonvanishing of the cohomology class. For the sake of complements, we reproduce the construction.

Suppose $a$ and $b$ are complex numbers such that the elements $(1,0),(0,1)$, $(a, 0)$ and $(0, b)$ form a basis for $\mathbf{C}^{2}$ over $\mathbf{R}$. Let $\Gamma \subset \mathbf{C}^{2}$ be the lattice generated by these elements over $\mathbf{Z}$, and let $\tilde{\Gamma}$ be the subgroup of $\Gamma$ generated by $(1,0),(0,1),(a, b)$ over $\mathbf{Z}$. Then $\mathbf{C}^{2} / \Gamma$ is an Abelian variety which is biholomorphic to a product of 1-dimensional tori, and the map

$$
\tilde{M}=\mathbf{C}^{2} / \tilde{\Gamma} \longrightarrow M=\mathbf{C}^{2} / \Gamma
$$

is a covering map. If $1, a$ and $b$ are linearly independent over $\mathbf{Z}$, then the only holomorphic functions on $\tilde{M}$ are the constants.

Now let $L$ be a positive holomorphic line bundle over $M$ and suppose that the cohomology class of $\pi^{*} L$ vanishes. Let $s$ be the holomorphic section of $\pi^{*} L$ without zeros as above. Since $\mathcal{O}(\tilde{M})=\mathbf{C}$, every holomorphic section $h$ of $\pi^{*} L$ over $\tilde{M}$ can be written as $h=$ c.s where $c \in \mathbf{C}$. By Corollary 4.3 in [N2], there exists a positive integer $p$ and a holomorphic section $\sigma$ of $\pi^{*} L^{p}$ on $\tilde{M}$ such that $\|\sigma\|$ is unbounded on any infinite subset without limit point in $\tilde{M}$. Therefore $\sigma=c . \otimes_{1}^{p} s$ which implies $\lim _{x \rightarrow \partial \tilde{M}}\|s\|=+\infty$. Hence the strongly Psh function $\phi=-\log \|s\|^{2}$ is bounded from above on $\tilde{M}$. Let $\pi_{1}: \mathbf{C}^{2} \rightarrow \tilde{M}$ be the universal covering, and define a strongly Psh function $\theta$ on $\mathbf{C}^{2}$ by

$$
\theta:=\phi o \pi_{1} .
$$

Since $\mathbf{C}^{2}$ is parabolic, the only Psh function bounded from above are the constants. This implies that $\|s\|=$ constante which contradicts the growth of $s$. Hence $\left[\pi^{*} L\right] \neq 0$ in $H^{1}\left(\tilde{M} \mathcal{O}^{*}\right)$ for every positive line bundle $L$ on $M$.

\section{References}

[D] J.P. Demailly, Estimations $L^{2}$ pour l'operateur $\bar{\partial}$ d'un fibré holomorphr semipositif au dessus d'une variété Kählerienne complète, Annales Sci. Ec. Norm. sup., 15 (1982), 457-511. 
[H] L. Hörmander, An introduction to complex analysis in several variables, North Holland, Third Edition, 1990.

[N1] T. Nappier, Covering spaces of families of compact Riemann surfaces, Math. Annalen, 294 (1992), 523-549.

[N2] - Convexity properties of covering of smooth projective varietes, Math. Annalen, 286 (1990), 433-479.

[S] C. Siegel, Analytic functions of several variables, Institute for Advanced Study, Princeton, 1949.

[Wa] K. Watanabe, Covering of a projective algebraic manifold, Pacific J. Math., 96 (1981), 243-246.

[We] R.O. Wells, Differential analysis on complex manifolds, GTM, Springer, New York, 1980 .

Received June 11, 1996. This research was supported by Moroccan Civil Service grant FSK-1.11.95-2.17033.

IBN TOFAIL UNIVERSITY

P.O. 135 Kenitra

MorocCo 